\title{
Transition from Ekman flow to Taylor vortex flow in superfluid helium
}

\author{
By KAREN L. HENDERSON ${ }^{1}$ AND \\ CARLO F. BARE N G H I \\ ${ }^{1}$ School of Mathematical Sciences, University of the West of England, \\ Bristol, BS16 1QY, UK. \\ e-mail: Karen.Henderson@uwe.ac.uk \\ ${ }^{2}$ School of Mathematics, University of Newcastle, \\ Newcastle upon Tyne, NE1 7RU, UK. \\ e-mail: C.F.Barenghi@ncl.ac.uk
}

(Received 9 November 2018)

By numerically computing the steady axisymmetric flow of helium II confined inside a finite aspect ratio Couette annulus, we determine the transition from Ekman flow to Taylor vortex flow as a function of temperature and aspect ratio. We find that the lowReynolds number flow is quite different to that of a classical fluid, particularly at lower temperatures. At high aspect ratio our results confirm the existing linear stability theory of the onset of Taylor vortices, which assumes infinitely long cylinders.

\section{The equations of motion of superfluid helium}

As the temperature is reduced through $T=T_{\lambda}=2.1768 \mathrm{~K}$, liquid helium undergoes a phase transition from helium I (a classical Navier-Stokes fluid) to helium II (a superfluid). The superfluid state persists through to absolute zero at vapour pressure. The motion of helium II is well described by the hydrodynamic two-fluid theory of Landau \& Tisza (1987). According to this theory, helium II comprises two perfectly mixed fluids, the viscous normal fluid and the inviscid superfluid, of densities $\rho^{n}$ and $\rho^{s}$ respectively. The total density of helium II, $\rho=\rho^{n}+\rho^{s}$, does not vary much with the temperature $T$, whereas $\rho^{n}$ and $\rho^{s}$ depend strongly on $T$. At absolute zero, $T=0 \mathrm{~K}$, the normal fluid component vanishes and helium II is entirely superfluid $\left(\rho^{n}=0\right)$, whilst at the lambda point, $T=T_{\lambda}$, the superfluid component is zero $\left(\rho^{s}=0\right)$ and helium II becomes helium I, which is a classical Navier-Stokes fluid. If helium II is rotated with angular velocity $\Omega$ greater than some small critical value, then vortex filaments appear in the superfluid (Donnelly, 1991). The circulation around each vortex filament is quantised, in that

$$
\oint_{C} \boldsymbol{v}^{s} \cdot \boldsymbol{d l}=\Gamma
$$

where $\boldsymbol{v}^{s}$ is the superfluid velocity field, $\Gamma=9.97 \times 10^{-4} \mathrm{~cm}^{2} \mathrm{sec}^{-1}$ is the quantum of circulation (the ratio of Plank's constant and the mass of one helium atom) and $C$ is an arbitrary integration path around the axis of the filament. When helium II rotates the vortices align themselves to the direction of rotation and form a regular configuration with areal density (number of vortex filaments crossing the unit area perpendicular to the direction of rotation) $N=2 \Omega / \Gamma$ (Feynman's rule).

The hydrodynamics of the superfluid state is an interesting topic per se, but it is 
worth mentioning that attention to this problem has additional motivations. The first arises from the engineering applications: helium is the only substance available in liquid form at temperatures near absolute zero, so it is important as a cryogenics coolant. Applications range from infrared detectors in space science to the cooling of superconducting magnets in particle physics. The second motivation comes from recent experimental developments in which the relation between classical and quantum turbulence is investigated (for example see Smith et al. 1993; Barenghi, Swanson \& Donnelly 1995).

The most generally accepted equations for modelling the macroscopic flow of helium II are the Hall-Vinen-Bekharevich-Khalatnikov (HVBK) equations which were derived by a number of people over the years (Hall \& Vinen 1956; Hall 1960; Bekharevich \& Khalatnikov 1965; Hills \& Robert 1977). These equations extend Landau's two-fluid model to take into account the presence of quantised vortex lines in the flow. The derivation of the equations is based on a continuum approximation, assuming a high density of vortex lines, all aligned roughly in the same direction. For such situations, the superfluid vorticity, which is discrete in nature, may be approximated as a continuum, resulting in an effective superfluid vorticity field $\boldsymbol{\omega}^{s}=\operatorname{curl} \boldsymbol{v}^{s}$. The isothermal, incompressible HVBK equations may be written as:

$$
\begin{gathered}
\frac{\partial \boldsymbol{v}^{n}}{\partial t}+\left(\boldsymbol{v}^{n} \cdot \boldsymbol{\nabla}\right) \boldsymbol{v}^{n}=-\boldsymbol{\nabla} p^{n}+\nu^{n} \nabla^{2} \boldsymbol{v}^{n}+\frac{\rho^{s}}{\rho} \boldsymbol{F}, \\
\frac{\partial \boldsymbol{v}^{s}}{\partial t}+\left(\boldsymbol{v}^{s} \cdot \boldsymbol{\nabla}\right) \boldsymbol{v}^{s}=-\boldsymbol{\nabla} p^{s}+\boldsymbol{T}-\frac{\rho^{n}}{\rho} \boldsymbol{F}, \\
\boldsymbol{\nabla} \cdot \boldsymbol{v}^{n}=0, \quad \boldsymbol{\nabla} \cdot \boldsymbol{v}^{s}=0,
\end{gathered}
$$

where $\boldsymbol{v}^{n}$ is the normal fluid velocity, $\nu^{n}$ is the kinematic viscosity of the normal fluid and $p^{n}, p^{s}$ are effective pressures $\left(\boldsymbol{\nabla} p^{s}=(1 / \rho) \boldsymbol{\nabla} p-\frac{1}{2}\left(\rho^{n} / \rho\right) \boldsymbol{\nabla}\left(\boldsymbol{v}^{n}-\boldsymbol{v}^{s}\right)^{2}\right.$ and $\boldsymbol{\nabla} p^{n}=$ $(1 / \rho) \boldsymbol{\nabla} p+\frac{1}{2}\left(\rho^{s} / \rho\right) \boldsymbol{\nabla}\left(\boldsymbol{v}^{n}-\boldsymbol{v}^{s}\right)^{2}$ where $p$ is the pressure). The mutual friction force may be written as

$$
\boldsymbol{F}=\frac{1}{2} B \widehat{\boldsymbol{\omega}}^{s} \times\left(\boldsymbol{\omega}^{s} \times\left(\boldsymbol{v}^{n}-\boldsymbol{v}^{s}-\nu^{s} \boldsymbol{\nabla} \times \widehat{\boldsymbol{\omega}}^{s}\right)\right)+\frac{1}{2} B^{\prime} \boldsymbol{\omega}^{s} \times\left(\boldsymbol{v}^{n}-\boldsymbol{v}^{s}-\nu^{s} \boldsymbol{\nabla} \times \widehat{\boldsymbol{\omega}}^{s}\right)
$$

with $\widehat{\boldsymbol{\omega}}^{s}=\boldsymbol{\omega}^{s} /\left|\boldsymbol{\omega}^{s}\right|$ the unit vector in the direction of superfluid vorticity and $B, B^{\prime}$ are the temperature-dependent mutual friction parameters (Barenghi, Donnelly \& Vinen 1983, Donnelly \& Barenghi 1998). This force is due to collisions between the normal fluid (mainly rotons, at temperatures relevant to most experiments) and vortex lines. The vortex tension force may be written as

$$
\boldsymbol{T}=-\nu^{s} \boldsymbol{\omega}^{s} \times\left(\boldsymbol{\nabla} \times \widehat{\boldsymbol{\omega}}^{s}\right),
$$

and reflects the energy per unit length in the vortex lines. The vortex tension parameter

$$
\nu^{s}=(\Gamma / 4 \pi) \log \left(b_{0} / a_{0}\right)
$$

has the same dimension as kinematic viscosity but physically it is very different: it represents the ability of a vortex line to oscillate due to vortex waves which can be excited on the vortex lines themselves. The quantity $b_{0}=\left(\left|\boldsymbol{\omega}^{s}\right| / \Gamma\right)^{-1 / 2}$ represents the intervortex spacing and $a_{0} \approx 10^{-8} \mathrm{~cm}$ is the radius of the superfluid vortex core.

\section{Motivation and aim of this work}

In this paper we apply the HVBK equations to model helium II in a finite aspect ratio Couette annulus, that is flow of fluid confined radially between two concentric rotating cylinders and axially between two fixed plates separated by a distance $H$. We consider 


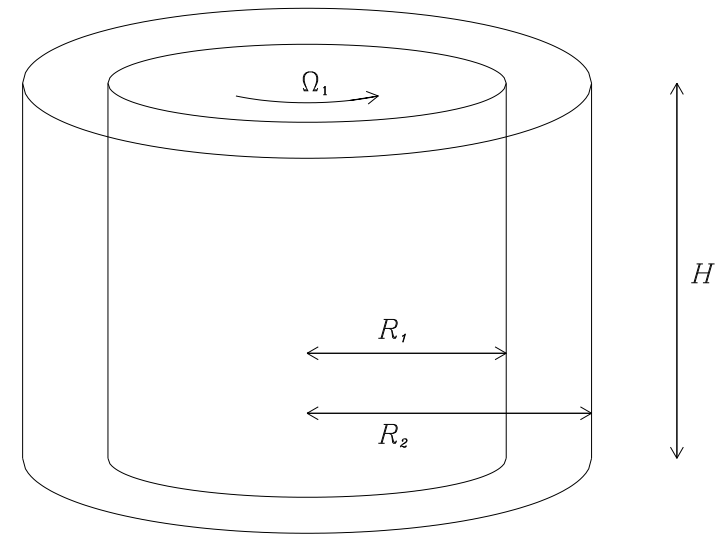

FIgURE 1. The problem's configuration. The fluid is contained inside a cylindrical box of inner radius $R_{1}$, outer radius $R_{2}$ and height $H$. The inner cylinder rotates at constant angular velocity $\Omega_{1}$, while the outer cylinder and the top and bottom end plate are stationary.

the inner cylinder $\left(r=R_{1}\right)$ to be rotating with angular velocity $\Omega_{1}$ and the outer cylinder $\left(r=R_{2}\right)$ to be fixed, see figure 1 Taylor-Couette flow has been used as a bench-mark for fluid mechanics since Taylor's pioneering work (1923) to investigate the transition from Couette flow to Taylor vortices, which established a firm ground for using the Navier-Stokes equations and the no-slip boundary conditions. Progress in helium II has been slower than for classical fluids due in part to problems of flow visualisation at such low temperatures. In considering a classical fluid, introduction of flakes or other small particles into the working fluid (usually oil or water), results in the Taylor vortices being clearly evident. In contrast there are only limited visualisation techniques available to the experimentalist at temperatures close to absolute zero. Recent attempts have been made to reveal the flow pattern of helium II by adding small particles (Bielert \& Stamm 1993). However this was only successful at high rotation rates (40 times the critical angular velocity at which linear stability analysis predicts Couette flow becomes unstable); in this regime the flow is turbulent and the validity of the HVBK equations is not clear. Clearly the lack of direct flow visualisation gives additional motivation to our work.

Experiments on helium II between concentric cylinders were first performed by Kapitza in 1941 and Donnelly \& LaMar (1988) have written a review of experiments involving helium II in Couette apparatus. Early Taylor-Couette experiments were concerned with determining the viscosity of helium II by measuring the torque exerted by the flow on the stationary cylinder. A break in the linear dependence of the torque with the angular velocity of the rotating cylinder is taken to denote a transition from one solution to another. A second experimental technique is available, that of measuring the extra attenuation of a second sound wave, which can be used to probe the superfluid vorticity. Second sound waves occur when there is a periodic counterflow between the normal fluid and superfluid, which corresponds to a wave of heat. Angular velocity is plotted against the attenuation factor and breaks in the curve are interpreted as transitions in the flow. By measuring the extra attenuation of second sound waves in the axial, azimuthal and radial directions it is theoretically possible to get an idea of the number and direction 
of the quantised vortex lines. In practice the information obtained is less complete than this.

Despite the fact that the HVBK equations were established in 1961, they have been used mainly to model helium II in a rotating container (solid body rotation). Unfortunately solid body rotation (which corresponds to a spatially uniform configuration of superfluid vortices) is not a strict test of the equations of motion because it is too simple; the terms in the HVBK equations which involve the vortex tension vanish. Only recently have the HVBK equations been validated for a non-trivial flow configuration: Couette flow. For the case of infinitely long cylinders, both superfluid and normal fluid velocity fields have the following Couette flow profile:

$$
\boldsymbol{v}^{n}=\boldsymbol{v}^{s}=\frac{\left(-\Omega_{1} R_{1}^{2} r+\Omega_{1} R_{1}^{2} R_{2}^{2} r^{-1}\right)}{\left(R_{2}^{2}-R_{1}^{2}\right)} \widehat{\boldsymbol{\phi}}
$$

where $(r, \phi, z)$ are cylindrical coordinates and $\widehat{\phi}$ is the unit vector in the azimuthal direction. After making the usual assumption of infinitely long cylinders, Barenghi \& Jones (1988) and Barenghi (1992) considered the linear stability of Couette flow with respect to infinitesimal perturbations of the form $\exp (\mathrm{i} m \phi+\mathrm{i} k z+\sigma t)$ where $\sigma$ is the complex growth rate, $m$ the azimuthal wavenumber and $k$ the dimensionless axial wavenumber (expressed in units of $1 / \delta$ where $\delta=R_{2}-R_{1}$ is the gap width). The driving parameter of the problem is the Reynolds number,

$$
R e=\frac{\Omega_{1} R_{1} \delta}{\nu^{n}},
$$

which represents the dimensionless velocity of the inner cylinder. Barenghi \& Jones (1988) found that if the inner cylinder rotates sufficiently fast, at a certain critical value $R \mathrm{e}=R \mathrm{e}_{\text {crit }}$ the growth rate, $\operatorname{Real}(\sigma)$ of the axisymmetric $(m=0)$ perturbation becomes positive, hence Couette flow becomes unstable. This transition corresponds to the onset of Taylor vortices for a classical fluid. Barenghi \& Jones (1988) also determined the temperature dependence of the critical Reynolds number, $R \mathrm{e}_{\text {crit }}$, and of the critical axial wavenumber, $k_{\text {crit }}$. Their work prompted further experiments and good agreement between the predicted and measured values of $R \mathrm{e}_{\text {crit }}$ was found (Barenghi 1992), particularly for temperatures close to the lambda point. The success of the linear stability analysis prompted further work, namely the study of nonlinear Taylor flow in infinitely long cylinders (Henderson, Barenghi \& Jones 1995). Comparisons with existing experimental data and numerical results further validated the HVBK model in the high temperature regime (Henderson \& Barenghi 1994).

Unfortunately at lower temperatures $(T<2 \mathrm{~K})$, although there was qualitative agreement, the measured critical Reynolds numbers were larger than those predicted by the linear stability analysis. In the classical Taylor-Couette problem the critical value of the dimensionless axial wavenumber is $k_{\text {crit }} \approx \pi$, hence each individual Taylor vortex cell is approximately square (the extension in the axial direction is equal to the gap's size). In the case of helium II, Barenghi \& Jones (1998) found that $k_{c r i t} \rightarrow \pi$ as $T \rightarrow T_{\lambda}$, as expected in the limit of a pure normal fluid. However, as the temperature is reduced, they found that $k_{\text {crit }}$ decreases and tends to zero in the limit of a pure superfluid. This result suggests that the discrepancy between theory and experiments for $T<2 \mathrm{~K}$ is due to end effects: there are not enough Taylor vortex cells in the typical experimental apparatus, thus the infinite cylinder assumption breaks down. A better theory is required and that is what we set out to do in this paper.

It is known in the classical Taylor-Couette literature that the presence of fixed ends induces a large scale Ekman circulation in which the fluid moves radially inward near the 
top and bottom ends of the Couette apparatus and moves radially outward in the middle. This effect is caused by the no-slip boundary conditions; the centrifugal force pushes the fluid outwards at the centreline, where the braking effect of the end plates is least, so the fluid near the end plates moves inwards to conserve mass. In the case of helium II the normal fluid obeys the same no-slip boundary condition of an ordinary classical fluid, but there are also superfluid boundary conditions to take into account. A discussion of what these superfluid boundary conditions should be is contained in our previous paper (Henderson \& Barenghi 2000), in which we also showed that the competition between the normal fluid and superfluid boundary conditions has unexpected effects on the direction of rotation of the Ekman circulation in a unit aspect ratio annulus. The aim of the current paper is to understand how the appearance of Taylor vortex flow is effected by the underlying Ekman circulation. Firstly we shall investigate the low Reynolds number flow of helium II at varying aspect ratios to see how it differs from the flow of a classical Navier-Stokes fluid. Secondly, we shall determine the transition from Ekman cells to Taylor vortices and compare the Taylor-Couette flow of helium II in an enclosed annulus to the flow in an infinite apparatus (Henderson et al. 1995). If, for sufficiently long cylinders, the results of the linear stability theory are recovered, contact will be made between theory and experiments at low temperatures.

\section{Model}

We consider the fluid to be confined radially between two concentric cylinders of inner and outer radius $R_{1}$ and $R_{2}$, and axially between two fixed plates which are separated by a distance $H$. The top and bottom plates and the outer cylinder are held stationary and the inner cylinder rotates at constant angular velocity $\Omega_{1}$. Throughout this work we shall consider the radius ratio, $\eta=R_{1} / R_{2}=0.976$ as in the experimental apparatus of Swanson \& Donnelly (1991) and will vary the Reynolds number, $R \mathrm{e}=\Omega_{1} R_{1} \delta / \nu^{n}$, the aspect ratio, $h=H / \delta$ and the temperature, $T$.

In order to solve the HVBK equations (1.2 1.4 we need boundary conditions. The boundary conditions for the normal fluid are no-slip, that is, working in cylindrical coordinates $\boldsymbol{v}^{n}=\Omega_{1} R_{1} \widehat{\boldsymbol{\phi}}$ at $r=R_{1}$ and $\boldsymbol{v}^{n}=0$ at $r=R_{2}, z=0, z=H$. The boundary conditions for the superfluid are more delicate. Given $\boldsymbol{n}$, a normal to the boundary, we have that $\boldsymbol{v}^{s} \cdot \boldsymbol{n}=0$ at $r=R_{1}, R_{2}$ and $z=0, H$, ensuring no flow normal to the boundaries. For the remaining conditions we have taken

$$
\boldsymbol{\omega}^{s} \times \widehat{\boldsymbol{z}}=0 \text { at } r=R_{1}, R_{2} \text { and } z=0, H,
$$

where $\widehat{z}$ is the unit vector in the axial direction. Thus the superfluid vorticity is taken to be purely axial at the boundaries. The condition on the cylinder walls, $r=R_{1}$ and $r=R_{2}$, has been discussed in a previous paper (Henderson et al. 1995) whilst the condition on the ends of the cylinder, $z=0$ and $z=H$, corresponds to perfect sliding of the vortex lines (Khalatnikov 1965). It must be stressed that as yet there is no experimental evidence for the boundary conditions which we propose; in fact, a motivation behind or work is to explore the consequence of assuming certain boundary conditions, hoping to stimulate experimental work on this issue. Other possibilities are that the vortex lines remain totally or partially pinned at the boundaries. Total pinning of the vortex lines on the ends of the cylinders cannot occur because each rotation would wrap up the vortex lines until de-pinning takes place. Partial sliding is a possibility, however this would introduce extra unknown parameters into the problem depending on the nature and the smoothness of the boundaries, so we have chosen to adopt perfect sliding.

We make the simplifying assumption that the flow is axisymmetric and introduce 
stream functions $\psi^{n}, \psi^{s}$ such that

$$
v_{r}^{p}=-\frac{1}{r} \frac{\partial \psi^{p}}{\partial z}, \quad v_{z}^{p}=\frac{1}{r} \frac{\partial \psi^{p}}{\partial r}
$$

where $p=n, s$ represents the normal fluid, superfluid respectively. Introducing the stream functions ensures that continuity (1.4) is automatically satisfied. A finite difference approach is used to obtain solutions for $\psi^{p}, v_{\phi}^{p}$ and $\omega_{\phi}^{p}$. The equations satisfied by these quantities are obtained by taking the $\phi$ component of (1.2 1.3) and the $\phi$ component of the curl of (1.21.3). A Poisson's equation links the stream function $\psi^{p}$ with the azimuthal component of vorticity $\omega_{\phi}^{p}$. These equations are solved on the computational domain $R_{1} \leq r \leq R_{2}, 0 \leq z \leq H / 2$ and symmetry is assumed to calculate the derivatives on the centreline $z=H / 2$. A uniform grid is used in both the radial and axial direction and the equations are stepped forward in time until a steady solution is achieved. The computational cost given our criterion of convergence determines the largest aspect ratio which we compute $(h=8)$. The equations are made dimensionless using the gap width $\delta$ as the unit of length and the normal fluid viscous time scale $\delta^{2} / \nu^{n}$ as the unit of time. For further details of the numerical method, see Henderson \& Barenghi (2000).

\section{Results}

In this section we refer to previous results (Henderson \& Barenghi 2000) in which we considered the low Reynolds number flow of helium II in a unit aspect ratio Couette annulus; this geometry constrains the flow to a simple Ekman circulation. The key discovery was the anomalous Ekman motion of helium II, compared to a classical Navier-Stokes fluid. We found that the pair of superfluid Ekman cells always rotate in a counter-classical direction (which means for example that $v_{r}^{s}<0$ at the centreline $z=H / 2$ ), whilst the normal fluid Ekman cells rotate classically $\left(v_{r}^{n}>0\right.$ at $\left.z=H / 2\right)$ at temperatures close to the lambda temperature, but reverse $\left(v_{r}^{n}<0\right.$ at $\left.z=H / 2\right)$ at lower temperatures. It was also found that the azimuthal superfluid velocity is almost independent of $z$. This effect becomes more pronounced at lower temperatures and was found to be due to the tension in the superfluid vortex lines.

In this paper, the parameters which are varied are the temperature $T$, Reynolds number $R$ e of the inner cylinder and aspect ratio $h$. Figures 246 show contour plots of the normal and superfluid stream functions, where the plots are shown for the complete cross-section of the annulus; $R_{1} \leq r \leq R_{2}, 0 \leq z \leq H$ with the inner/outer cylinder on the left/right respectively. The maximum value of each field is printed underneath the corresponding contour plot. Light and dark regions correspond to positive and negative contour lines respectively.

In figure 2 we investigate how varying the aspect ratio effects the flow of helium II at $T=2.11 \mathrm{~K}$ and $R \mathrm{e}=100$. This Reynolds number is appreciably below the critical Reynolds number $\left(R \mathrm{e}_{\text {crit }}=355\right)$ at which the linear stability analysis predicts Couette flow becomes unstable in the infinite cylinder approximation. It is apparent from figure 2 $(a)$ that since the normal fluid and superfluid stream functions increase with $z$ across the centreline $z=H / 2$, (3.2 $a)$ predicts that the radial velocity components are negative at the centreline, in contrast to the motion of a classical Navier-Stokes fluid. Thus for small aspect ratios $(h \leq 2.5)$ both the normal fluid and superfluid rotate counter-classically in a pair of matching Ekman cells. This behaviour has been reported previously (Henderson \& Barenghi 2000) and is due to the boundary conditions satisfied by the vortex lines on the top and bottom of the cylinders. Figure 2 also shows that the superfluid Ekman cells fill the whole annulus, becoming more elongated as the aspect 


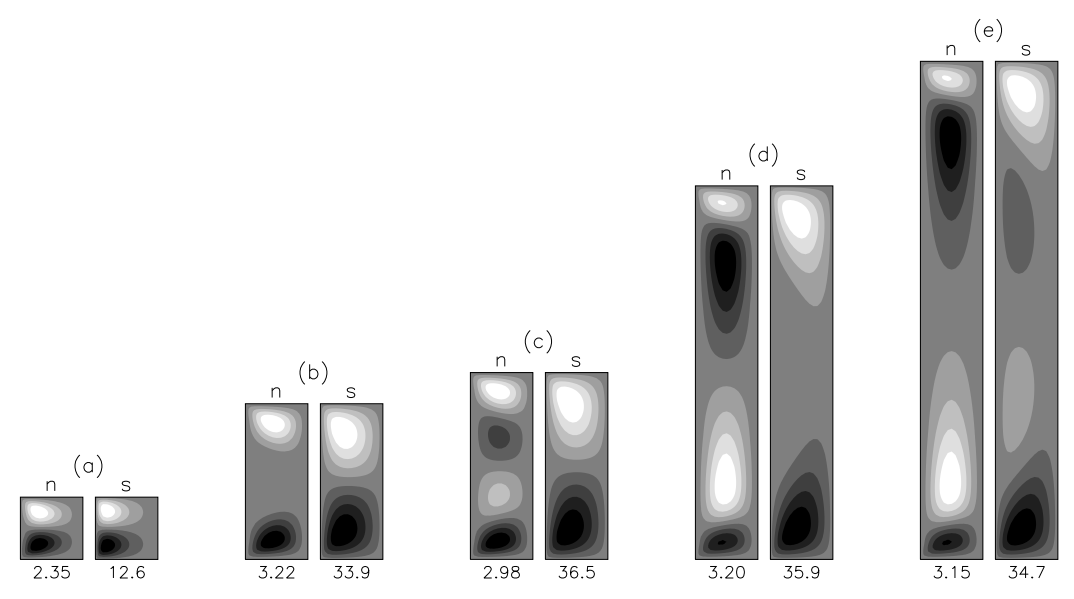

Figure 2. Contour plots of the stream function of the normal fluid (n) and the superfluid (s) at $T=2.11 \mathrm{~K}$ and $R \mathrm{e}=100$ at aspect ratios: (a) $h=1$, (b) $h=2.5$, (c) $h=3$, (d) $h=6$, (e) $h=8$.

ratio is increased. In contrast, the normal fluid Ekman cells, tend to retain their size and are positioned towards the ends of the cylinders.

As the aspect ratio is increased further $(h>2.5)$ the superfluid remains in the form of two counter-rotating cells whilst the normal fluid splits into four cells with the cells adjacent to the ends of the cylinder rotating in the same direction as the superfluid. The inner cells of the normal fluid strengthen and fill the annulus as the aspect ratio is increased. At aspect ratio $h=8$ the superfluid splits into four cells and although the size of the cells is different to those of the normal fluid, the direction of rotation is the same for both fluids.

Taken together these results demonstrate that the low-Reynolds number flow of helium II is dominated by end effects, which force the outer cells of the normal and superfluid to rotate counter-classically. As the aspect ratio is increased the influence of the ends at the centre of the apparatus becomes weaker and classical behaviour is observed in both fluids, with outflow at the centreline, $z=H / 2$. In our previous work (Henderson \& Barenghi 2000) we highlighted the columnar motion of the azimuthal component of superfluid velocity. We find that as the aspect ratio is increased this column like motion becomes less pronounced.

In figure 3 we show how varying the temperature effects the flow of helium II at $R \mathrm{e}=$ 100 and $h=4$. At this aspect ratio, the maximum axial wavelength of a disturbance is, $\lambda_{\max }=4$ giving a corresponding minimum admissible axial wavenumber of $k_{\min }=\pi / 2$. Given this, the Reynolds number, $R \mathrm{e}=100$ is below the critical value predicted by linear stability analysis at which Couette flow become unstable for all temperatures considered. At temperatures close to the lambda temperature $(T \geq 2.16 \mathrm{~K})$ the normal fluid and superfluid rotate in a pair of Ekman cells. The normal fluid rotates in a classical direction and the superfluid rotates counter-classically. As the temperature is reduced the normal fluid splits into four cells, whilst the superfluid remains as two cells. In this region, the normal fluid cells closest to the ends of the cylinder rotate in the same direction as the superfluid and become stronger in magnitude and also fill more of the annulus as the temperature is reduced. The columnar behaviour of the azimuthal superfluid 

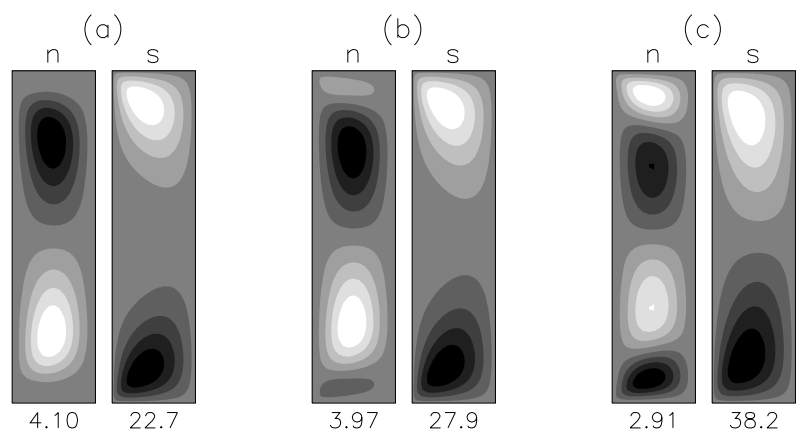

Figure 3. Contour plots of the stream function of the normal fluid (n) and the superfluid (s) at $R \mathrm{e}=100$ and $h=4$ at temperatures: (a) $T=2.16 \mathrm{~K}$, (b) $T=2.15 \mathrm{~K}$, (c) $T=2.1 \mathrm{~K}$.
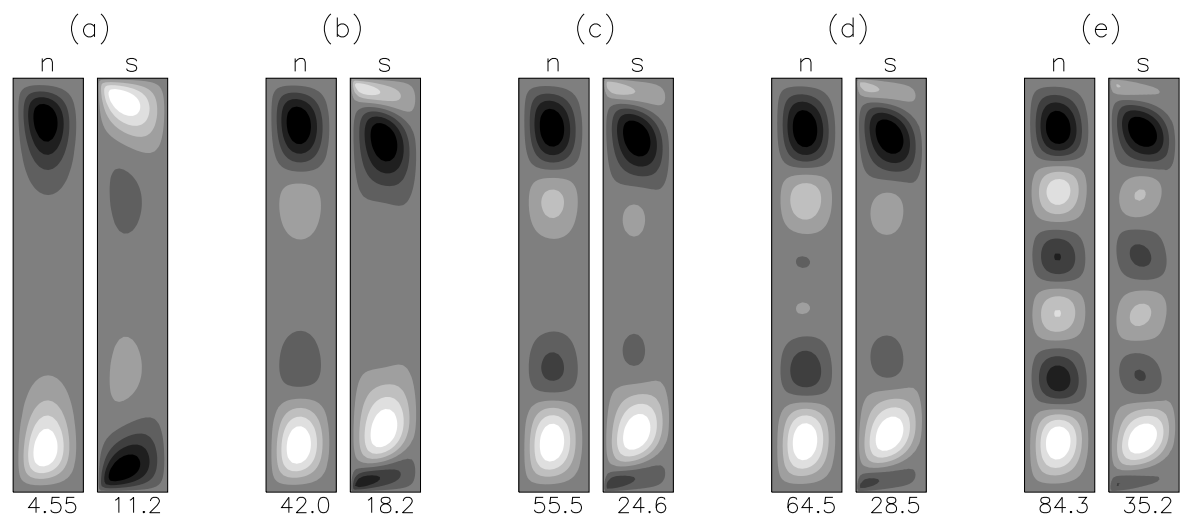

FiguRE 4. Contour plots of the stream function of the normal fluid (n) and the superfluid (s) at $T=2.17 \mathrm{~K}$ and $h=6$ at Reynolds numbers: (a) $R \mathrm{e}=100$, (b) $R \mathrm{e}=250$, (c) $R \mathrm{e}=270$, (d) $R \mathrm{e}=280$, (e) $R \mathrm{e}=300$.

velocity is not evident at temperatures close to the lambda temperature but becomes more pronounced as the temperature is reduced.

In figure 4 we investigate the transition from Ekman flow to Taylor vortex flow at $T=2.17 \mathrm{~K}$ and $h=6$. At low Reynolds numbers $(R \mathrm{e}=100)$ the normal fluid consists of a pair of classically rotating Ekman cells. The superfluid has four cells, with the cells adjacent to the ends of the cylinders rotating counter-classically, as predicted by the boundary conditions. As the Reynolds number is increased both the normal fluid and the superfluid develop more cells, with the cells closest to the ends rotating classically and counter-classically for the normal and superfluid respectively, as a consequence of the boundary conditions. At $R \mathrm{e}=300$ Taylor vortex flow is fully developed and we can see that despite the superfluid having an additional weak pair of cells close to the ends of the cylinders, the two fluids appear to match each other. In figure[5 we plot the centreline average values of the radial components of the normal fluid (solid) and superfluid (dashed) against Reynolds number. From the graph we can see that the transition to Taylor vortices occurs at approximately $R \mathrm{e}=275$, whilst stability analysis in the infinitely long cylinders approximation predicts $R \mathrm{e}_{\text {crit }}=278.7$ at $k_{\text {crit }}=2.9$.

In figure [6] we repeat the calculation at $h=6$ but setting a lower temperature, $T=2.16 \mathrm{~K}$. At low Reynolds numbers $(R \mathrm{e}=100)$ the normal fluid has a pair of 


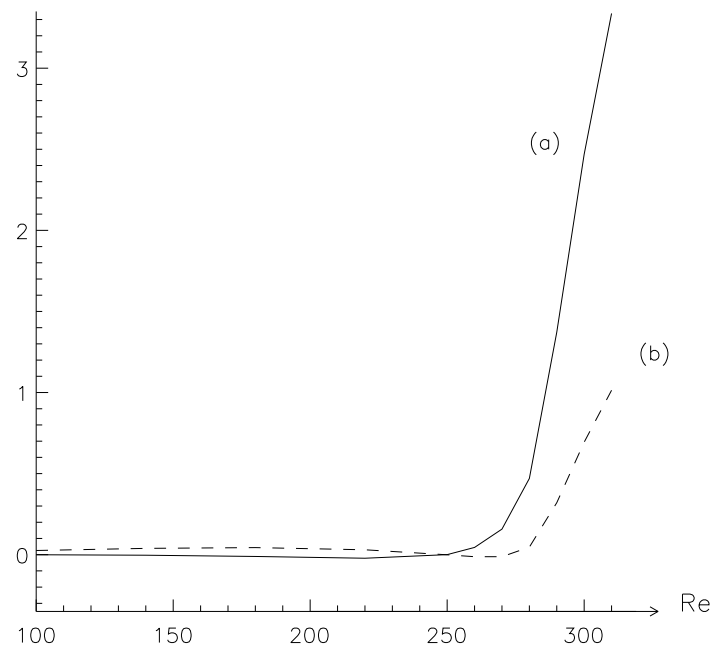

FiguRE 5. Plot of the average values of (a) $v_{r}^{n}$ (solid) and (b) $v_{r}^{s}$ (dashed) at the centreline $z=H / 2$ against Reynolds number.
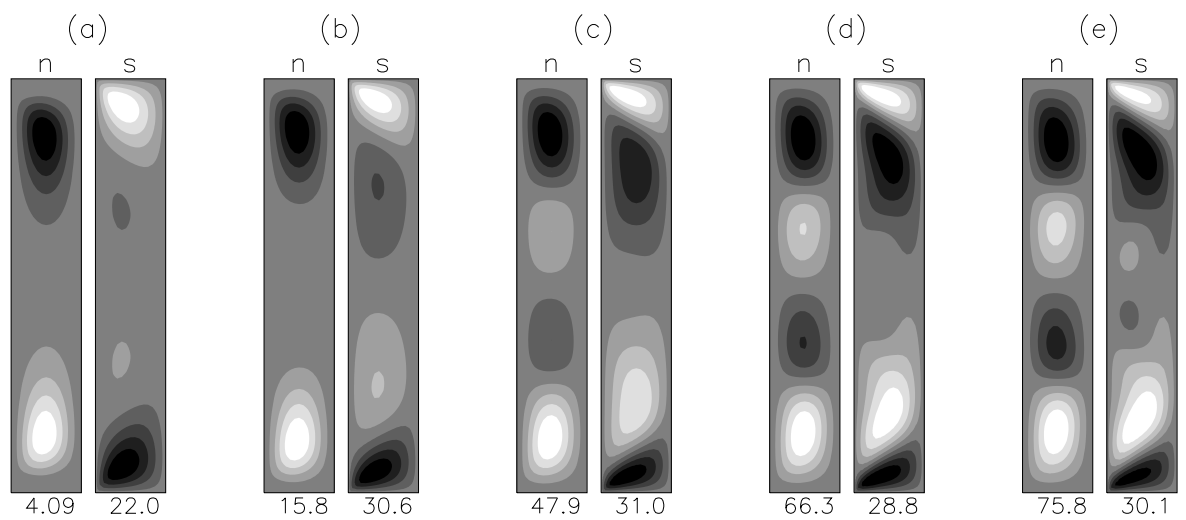

FiguRE 6. Contour plots of the stream function of the normal fluid (n) and the superfluid (s) at $T=2.16 \mathrm{~K}$ and $h=6$ at Reynolds numbers: (a) $R \mathrm{e}=100$, (b) $R \mathrm{e}=180$, (c) $R \mathrm{e}=275$, (d) $R \mathrm{e}=300,(\mathrm{e}) \operatorname{Re}=310$.

classically rotating Ekman cells. As before, the superfluid has four cells, and the cells which are adjacent to the ends of the cylinders rotate counter-classically, as predicted by the boundary conditions. As the Reynolds number is increased, the normal fluid develops four cells which fill the whole apparatus, not six as at $T=2.17 \mathrm{~K}$. The outer cells rotate classically due to the boundary conditions. The superfluid remains as four cells up to $R \mathrm{e}=300$, with the outer cells rotating counter-classically and the inner cells strengthing with increasing Reynolds number. At $R \mathrm{e}=310$ the superfluid develops an additional small weak pair of cells at the centre of the apparatus.

In figure 7 we plot the centreline average values of the radial components of the normal fluid (solid) and superfluid (dashed) against Reynolds number. The transition from the Ekman flow to the Taylor vortex flow is harder to distinguish than at higher temperatures and is slightly different for the normal and superfluid. It occurs at approximately $R \mathrm{e}=$ 270. What is interesting about these results is that the Taylor vortices for the normal fluid 


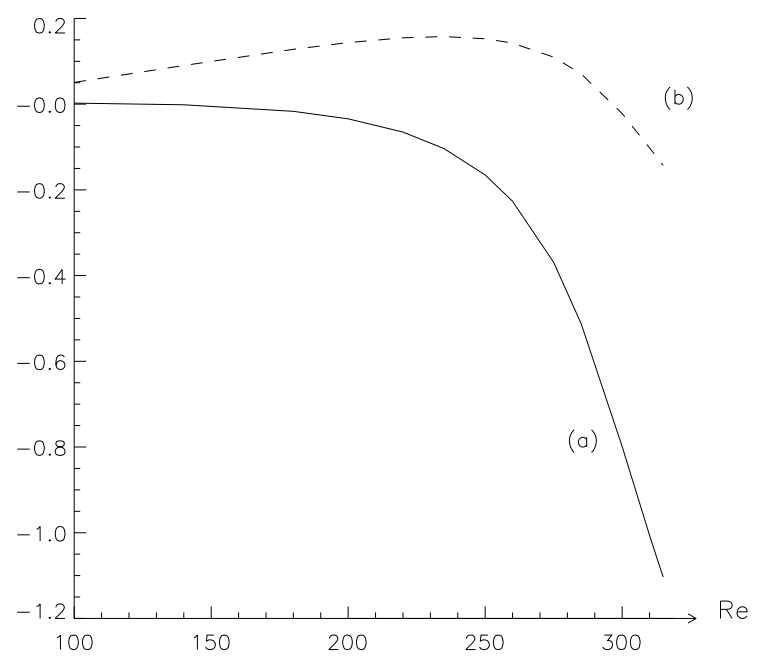

FiguRE 7. Plot of the average values of (a) $v_{r}^{n}$ (solid) and (b) $v_{r}^{s}$ (dashed) at the centreline $z=H / 2$ against Reynolds number.

are elongated, being $1 \frac{1}{2}$ times longer than they are wide. This is consistent with the linear stability analysis which predicts that $R \mathrm{e}_{\text {crit }}=310.68$ at the critical axial wavenumber of $k_{\text {crit }}=2.35$. The elongation of the Taylor cells was first predicted by Barenghi \& Jones (1988) and this is the first time that it has been observed numerically taking end effects into account.

We find that in general the superfluid vorticity is primarily axial and concentrated towards the ends of the cylinders close to the inner cylinder. This predicted result could be observed experimentally by measuring the extra attenuation of second sound waves due to the vortex lines at the centre and ends of the apparatus. As the Reynolds number is increased, there is a much larger deflection of the vortex lines in the radial direction. This deflection has been observed experimentally in the transition to Taylor vortices (Swanson \& Donnelly 1991). In figure 8 we plot the superfluid vorticity components of helium II at $T=2.16 \mathrm{~K}, h=6$ at the Reynolds numbers (a) $R \mathrm{e}=100$ and (b) $R \mathrm{e}=300$ for the middle two-thirds of the apparatus $(1 \leq z / \delta \leq 5)$. This enables us to see what happens to the vortex lines in the centre of the apparatus away from the cylinder ends, where there is stronger shear which would make the contour lines in the middle of the apparatus less visible. The deflection in the radial direction is clearly visible for $R \mathrm{e}=300$. In the Taylor vortex flow regime, the vorticity pattern is similar to that found when considering the Taylor-Couette flow of helium II without end effects (Henderson et al. 1995).

\section{Conclusions}

By solving the nonlinear HVBK equations in a finite aspect ratio configuration we have determined how the fixed ends of the cylinders effect the Ekman flow and the transition to Taylor vortex flow.

For low-Reynolds number flow, the main result is the anomalous Ekman circulation of helium II when compared to that of a classical Navier-Stokes fluid. At short aspect ratios both normal fluid and superfluid rotate as a pair of Ekman cells. The superfluid cells always rotate counter-classically, whilst the sense of rotation of the normal fluid is temperature dependent. At larger aspect ratios the influence of the ends of the cylinders 

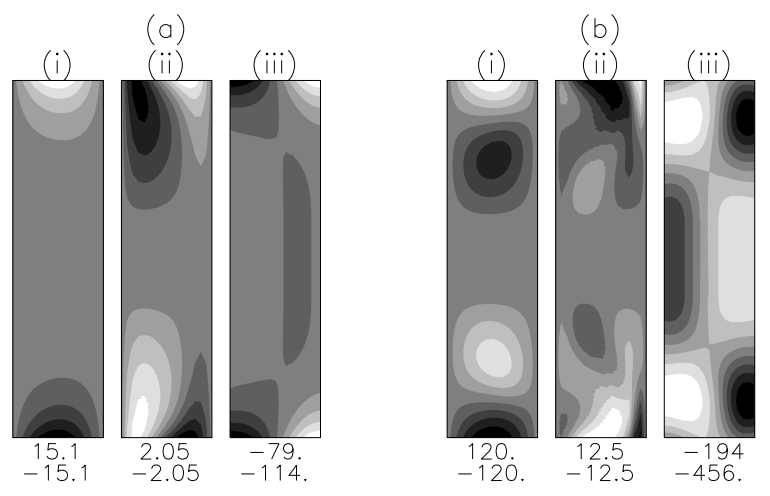

Figure 8. Contour plots of the components of superfluid vorticity: (i) $\omega_{r}^{s}$, (ii) $\omega_{\phi}^{s}$, (iii) $\omega_{z}^{s}$ at $T=2.16 \mathrm{~K}, h=6$ for (a) $R \mathrm{e}=100$, (b) $R \mathrm{e}=300$ in the middle two-thirds of the annulus, $1 \leq z / \delta \leq 5$. The maximum and minimum values of each field are printed underneath the corresponding plot.

towards the centre of the apparatus diminishes and at lower temperatures the normal fluid develops additional cells resulting in classical outflow at the centreline. The superfluid also develops extra cells to match the flow of the normal fluid at the centre.

We have found that at $T=2.17 \mathrm{~K}$ and aspect ratio as small as $h=6$, in the regions way from the ends of the cylinders, the superfluid's pattern matches that of the normal fluid. The transition to Taylor vortex flow occurs at a Reynolds number close to that predicted by the linear stability analysis in the infinite cylinder approximation. At the slightly lower temperature of $T=2.16 \mathrm{~K}$ we find that the normal fluid develops elongated Taylor cells, as predicted by the linear stability analysis. The superfluid flow pattern is similar to that of the normal fluid away from the ends of the cylinders but the matching is not as pronounced as at higher temperatures.

Finally we note that gaining more insight into the flow of helium II is particularly worthwhile because of the lack of direct flow visualisation at temperature close to absolute zero.

\section{REFERENCES}

Barenghi, C. F. 1992 Vortices and the Couette flow of helium II. Physical Review B 45, 2290-2293.

Barenghi, C. F., Donnelly, R. J. \& Vinen, W. F. 1983 Friction on quantized vortices in He II. A review. Journal of Low Temperature Physics 52, 189-247.

BAREnghi, C. F. \& Jones, C. A. 1988 The stability of the Couette flow of helium II. Journal of Fluid Mechanics 197, 551-569.

Barenghi, C. F., Swanson, C. J. \& Donnelly, R. J. 1995 Emerging issues in helium turbulence. Journal of Low Temperature Physics 100, 385-413.

Bekharevich, I. L. \& Khalatnikov, I. M. 1961 Phenomenological derivation of the equations of vortex motion in He II. Soviet Phys. JETP 13, 643-646.

Bielert, F. \& Stamm, G. 1993 Visualisation of Taylor Couette flow in superfluid helium. Cryogenics 33, 938-940.

Donnelly, R. J. 1991 Quantized Vortex Lines in Helium II. Cambridge University Press.

Donnelly, R. J. \& Barenghi, C. F. 1998 The observed properties of liquid helium at the saturated vapour pressure. J. Physical and Chemical Reference Data 27, 1217-1274.

Donnelly, R. J. \& Lamar, M. M. 1988 Flow and stability of helium II between rotating cylinders. Journal of Fluid Mechanics 186, 163-198. 
Feynman, R. P. 1955 Application of quantum mechanics to liquid helium. In Progress in Low Temperature Physics, , vol. 1. C. J. Gorter, North Holland.

Hall, H. E. 1960 The rotation of liquid helium II. Advances in Physics 9, 89-146.

Hall, H. E. \& Vinen, W. F. 1956 The rotation of liquid He II. II the theory of mutual friction in uniformly rotating He II. Proceedings of the Royal Society of London A 238, 215-234.

Henderson, K. L. \& BArenghi, C. F. 1994 Calculation of the torque in nonlinear Taylor vortex flow of helium II. Physics Letters A 191, 438-442.

Henderson, K. L. \& BAREnGhi, C. F. 2000 The anomalous motion of superfluid helium in a rotating cavity. Journal of Fluid Mechanics 406, 199-219.

Henderson, K. L., Barenghi, C. F. \& Jones, C. 1995 Nonlinear Taylor-Couette flow of helium II. Journal of Fluid Mechanics 283, 329-340.

Hills, R. N. \& Roberts, P. H. 1977 Superfluid mechanics for a high density of vortex lines. Arch Rat Mech Anal 66, 43-71.

KAPITZA, P. L. 1941 Journal of Physics, USSR 4, 181.

Khalatnikov, I. M. 1965 An Introduction to the Theory Superfluidity. Benjamin.

Landau, L. D. \& Lifshitz, E. M. 1987 Fluid Mechanics. Pergamon.

Smith, M. R., Donnelly, R. J., Goldenfeld, N. \& Vinen, W. F. 1993 Decay of vorticity in homogeneous turbulence. Physical Review Letters 71, 2583-2586.

Swanson, C. J. \& Donnelly, R. J. 1991 Instability of Taylor-Couette flow of helium II. Physical Review Letters 67, 1578-1581.

TAYLOR, G. I. 1923 Stability of a viscous liquid contained between two rotating cylinders. Phil. Trans. Roy. Soc. Lond. A 223, 289-343. 